\title{
ON A LOCALIZED RIEMANNIAN PENROSE INEQUALITY
}

\author{
PENGZI MIAO
}

\begin{abstract}
Let $\Omega$ be a compact, orientable, three dimensional Riemannian manifold with boundary with nonnegative scalar curvature. Suppose its boundary $\partial \Omega$ is the disjoint union of two pieces: $\Sigma_{H}$ and $\Sigma_{O}$, where $\Sigma_{H}$ consists of the unique closed minimal surfaces in $\Omega$ and $\Sigma_{O}$ is metrically a round sphere. We obtain an inequality relating the area of $\Sigma_{H}$ to the area and the total mean curvature of $\Sigma_{O}$. Such an $\Omega$ may be thought as a region, surrounding the outermost apparent horizons of black holes, in a time-symmetric slice of a space-time in the context of general relativity. The inequality we establish has close ties with the Riemannian Penrose Inequality, proved by Huisken and Ilmanen [9] and by Bray [5].
\end{abstract}

\section{INTRODUCTION}

Let $M$ be a complete, asymptotically flat 3-manifold with nonnegative scalar curvature. Suppose its boundary $\partial M$ consists of the outermost minimal surfaces in $M$. The Riemannian Penrose Inequality, first proved by Huisken and Ilmanen [9] for a connected $\partial M$, and then by Bray [5] for $\partial M$ with any number of components, states that

$$
m_{A D M}(M) \geq \sqrt{\frac{A}{16 \pi}}
$$

where $m_{A D M}(M)$ is the ADM mass [1] of $M$ and $A$ is the area of $\partial M$. Furthermore, the equality holds if and only if $M$ is isometric to a spatial Schwarzschild manifold outside its horizon.

Motivated by the quasi-local mass question in general relativity (see [2], [6], [7], etc.), we would like to seek a localized statement of the above inequality (11). To be precise, we are interested in a compact, orientable, 3-dimensional Riemannian manifold $\Omega$ with boundary. We call $\Omega$ a body surrounding horizons if its boundary $\partial \Omega$ is the disjoint union of two pieces: $\Sigma_{O}$ (the outer boundary) and $\Sigma_{H}$ (the horizon boundary), and $\Omega$ satisfies the following assumptions:

This research is supported in part by the Australian Research Council. 
(a) $\Sigma_{O}$ is topologically a 2 -sphere.

(b) Each component of $\Sigma_{H}$ is a minimal surface in $\Omega$.

(c) There are no other closed minimal surfaces in $\Omega$.

Physically, $\Omega$ is to be thought as a finite region in a time-symmetric slice of a spacetime containing black holes and $\Sigma_{H}$ corresponds to the outermost apparent horizon of the black holes. In such a context, if the spacetime satisfies the dominant energy condition and $m_{Q M}\left(\Sigma_{O}\right)$ represents some quantity (to be defined) which could measure the quasi-local mass of $\Sigma_{O}$, then one would expect

$$
m_{Q M}\left(\Sigma_{O}\right) \geq \sqrt{\frac{A}{16 \pi}} .
$$

In this paper, we are able to establish an equality of the above form for a special class of body surrounding horizons. Our main result is

Theorem 1. Let $\Omega$ be a body surrounding horizons whose outer boundary $\Sigma_{O}$ is metrically a round sphere. Suppose $\Omega$ has nonnegative scalar curvature and $\Sigma_{O}$ has positive mean curvature. Then

$$
m\left(\Sigma_{O}\right) \geq \sqrt{\frac{\left|\Sigma_{H}\right|}{16 \pi}}
$$

where $m\left(\Sigma_{O}\right)$ is defined by

$$
m\left(\Sigma_{O}\right)=\sqrt{\frac{\left|\Sigma_{O}\right|}{16 \pi}}\left[1-\frac{1}{16 \pi\left|\Sigma_{O}\right|}\left(\oint_{\Sigma_{O}} H d \sigma\right)^{2}\right],
$$

where $\left|\Sigma_{H}\right|,\left|\Sigma_{O}\right|$ are the area of $\Sigma_{H}, \Sigma_{O}, H$ is the mean curvature of $\Sigma_{O}$ (with respect to the outward normal) in $\Omega$, and $d \sigma$ is the surface measure of the induced metric. When equality holds, $\Sigma_{O}$ is a surface with constant mean curvature.

We remark that, assuming (3) in Theorem 1 holds in the first place, one can derive (11) in the Riemannian Penrose Inequality. That is because, by a result of Bray [4, to prove (11), one suffices to prove it for a special asymptotically flat manifold $M$ which, outside some compact set $K$, is isometric to a spatial Schwarzschild manifold near infinity. On such an $M$, let $\Omega$ be a compact region containing $K$ such that its outer boundary $\Sigma_{O}$ is a rotationally symmetric sphere in the Schwarzschild region. Applying Theorem 1 to such an $\Omega$ and observing that, in this case, the quantity $m\left(\Sigma_{O}\right)$ coincides with the Hawking quasi-local mass [8] of $\Sigma_{O}$, hence agrees with the ADM mass of $M$, we see that (3) implies (11). On the other hand, our proof of Theorem 1 does make critical use of (1). Therefore, (3) and (1) are equivalent. 
Besides the Riemannian Penrose Inequality, Theorem 1 is also largely inspired by the following result of Shi and Tam [14]:

Theorem 2. (Shi-Tam) Let $\tilde{\Omega}$ be a compact, 3-dimensional Riemannian manifold with boundary with nonnegative scalar curvature. Suppose $\partial \tilde{\Omega}$ has finitely many components $\Sigma_{i}$ so that each $\Sigma_{i}$ has positive Gaussian curvature and positive mean curvature $H$ (with respect to the outward normal), then

$$
\oint_{\Sigma_{i}} H d \sigma \leq \oint_{\Sigma_{i}} H_{0} d \sigma
$$

where $H_{0}$ is the mean curvature of $\Sigma_{i}$ (with respect to the outward normal) when it is isometrically imbedded in $\mathbb{R}^{3}$. Furthermore, equality holds if and only if $\partial \tilde{\Omega}$ has only one component and $\tilde{\Omega}$ is isometric to a domain in $\mathbb{R}^{3}$.

Let $\tilde{\Omega}$ be given in Theorem 2 . Suppose $\partial \tilde{\Omega}$ has a component $\Sigma$ which is isometric to a round sphere with area $4 \pi R^{2}$, then

$$
\oint_{\Sigma} H_{0} d \sigma=8 \pi R
$$

and (5) yields

$$
\frac{1}{8 \pi} \oint_{\Sigma} H d \sigma \leq R
$$

Now suppose there is a closed minimal surfaces $\Sigma_{h}$ in $\tilde{\Omega}$ such that $\Sigma_{h}$ and $\Sigma$ bounds a region $\Omega$ which contains no other closed minimal surfaces in $\tilde{\Omega}$ (by minimizing area over surfaces homologous to $\Sigma$, such a $\Sigma_{h}$ always exists if $\partial \tilde{\Omega}$ has more than one components). Applying Theorem 1 to $\Omega$, we have

$$
\sqrt{\frac{\left|\Sigma_{h}\right|}{16 \pi}} \leq \sqrt{\frac{|\Sigma|}{16 \pi}}\left[1-\frac{1}{16 \pi|\Sigma|}\left(\oint_{\Sigma} H d \sigma\right)^{2}\right],
$$

which can be equivalently written as

$$
\frac{1}{8 \pi} \oint_{\Sigma} H d \sigma \leq \sqrt{R\left(R-R_{h}\right)}
$$

where $R=\sqrt{\frac{|\Sigma|}{4 \pi}}$ and $R_{h}=\sqrt{\frac{\left|\Sigma_{h}\right|}{4 \pi}}$. Therefore, Theorem 11 may be viewed as a refinement of Theorem 2 in this special case to include the effect on $\Sigma$ by the closed minimal surface in $\widetilde{\Omega}$ that lies "closest" to $\Sigma$.

In general relativity, Theorem 2 is a statement on the positivity of the Brown-York quasi-local mass $m_{B Y}(\partial \tilde{\Omega})[6$. Using the technique of weak inverse mean curvature flow developed by Huisken and Ilmanen 
[9], Shi and Tam [15 further proved that $m_{B Y}(\partial \tilde{\Omega})$ is bounded from below by the Hawking quasi-local mass $m_{H}(\partial \tilde{\Omega})$. Suggested by the quantity $m\left(\Sigma_{O}\right)$ in Theorem 1, we find some new geometric quantities associated to $\partial \tilde{\Omega}$, which are interestingly between $m_{B Y}(\partial \tilde{\Omega})$ and $m_{H}(\partial \tilde{\Omega})$ (hence providing another proof of $m_{B Y}(\partial \tilde{\Omega}) \geq m_{H}(\partial \tilde{\Omega})$.) We include this discussion at the end of the paper.

This paper is organized as follows. In Section 2, we review the approach of Shi and Tam in 14 since it plays a key role in our derivation of Theorem 1. The detailed proof of Theorem 1 is given in Section 3 , In Section 3.1, we establish a partially generalized Shi-Tam monotonicity. In Section 3.2, we make use of the Riemannian Penrose Inequality. In Section 4, we give some discussion on quasi-local mass. In particular, we introduce two quantities motivated by Theorem 1 and compare them with the Brown-York quasi-local mass $m_{B Y}(\Sigma)$ and the Hawking quasi-local mass $m_{H}(\Sigma)$.

Acknowledgement: The author wants to thank professor Hubert Bray for the helpful discussion leading to Section 4 .

\section{REVIEW OF SHI-TAM'S APPROACH}

In [14, Shi and Tam pioneered the idea of using results on asymptotically flat manifolds to study compact manifolds with boundary. We briefly review their approach in this section.

Let $\tilde{\Omega}$ be given in Theorem 2 . For simplicity, we assume $\partial \tilde{\Omega}$ has only one component $\Sigma$. Since $\Sigma$ has positive Gaussian curvature, $\Sigma$ can be isometrically imbedded in $\mathbb{R}^{3}$ as a strictly convex surface [11]. On the region $E$ exterior to $\Sigma$, the Euclidean metric $g_{0}$ can be written as

$$
g_{0}=d \rho^{2}+g_{\rho},
$$

where $g_{\rho}$ is the induced metric on each level set $\Sigma_{\rho}$ of the Euclidean distance function $\rho$ to $\Sigma$. Motivated by the quasi-spherical metric construction of Bartnik [3], Shi and Tam showed that there exists a positive function $u$ defined on $E$ such that the warped metric

$$
g_{u}=u^{2} d \rho^{2}+g_{\rho}
$$

has zero scalar curvature, is asymptotically flat and the mean curvature of $\Sigma$ in $\left(E, g_{u}\right)$ (with respect to the $\infty$-pointing normal) agrees with the mean curvature of $\Sigma$ in $\Omega$. Furthermore, as a key ingredient to prove their result, they showed that the quantity

$$
\oint_{\Sigma_{\rho}}\left(H_{0}-H_{u}\right) d \sigma
$$


is monotone non-increasing in $\rho$, and

$$
\lim _{\rho \rightarrow \infty} \oint_{\Sigma_{\rho}}\left(H_{0}-H_{u}\right) d \sigma=8 \pi m_{A D M}\left(g_{u}\right),
$$

where $H_{0}, H_{u}$ are the mean curvature of $\Sigma_{\rho}$ with respect to $g_{0}, g_{u}$, and $m_{A D M}\left(g_{u}\right)$ is the ADM mass of $g_{u}$. Let $M$ be the Riemannian manifold obtained by gluing $\left(E, g_{u}^{m}\right)$ to $\tilde{\Omega}$ along $\Sigma$. The metric on $M$ is asymptotically flat, has nonnegative scalar curvature away from $\Sigma$, is Lipschitz near $\Sigma$, and the mean curvatures of $\Sigma$ computed in both sides of $\Sigma$ in $M$ (with respect to the $\infty$-pointing normal) are the same. By generalizing Witten's spinor argument [16], Shi and Tam proved that the positive mass theorem [13] [16] remains valid on $M$ (see [10] for a non-spinor proof). Therefore,

$$
\oint_{\Sigma}\left(H_{0}-H_{u}\right) d \sigma \geq \lim _{\rho \rightarrow \infty} \oint_{\Sigma_{\rho}}\left(H_{0}-H_{u}\right) d \sigma=8 \pi m_{A D M}\left(g_{u}\right) \geq 0,
$$

with $\oint_{\Sigma}\left(H_{0}-H\right) d \sigma=0$ if and only if $H=H_{0}$ and $\tilde{\Omega}$ is isometric to a domain in $\mathbb{R}^{3}$.

\section{Proof of Theorem 1}

We are now in a position to prove Theorem 1. The basic idea is to deform the exterior region of a rotationally symmetric sphere in a spatial Schwarzschild manifold in a similar way as Shi and Tam did on $\mathbb{R}^{3}$, then attach it to a body surrounding horizons and apply the Riemannian Penrose Inequality to the gluing manifold. The key ingredient in our proof is the discovery of a new monotone quantity associated to the deformed metric. We divide the proof into two subsections.

\subsection{A monotonicity property for quasi-spherical metrics on a} Schwarzschild background. Consider part of a spatial Schwarzschild manifold

$$
\left(M_{r_{0}}^{m}, g^{m}\right)=\left(S^{2} \times\left[r_{0}, \infty\right), \frac{1}{1-\frac{2 m}{r}} d r^{2}+r^{2} d \sigma^{2}\right),
$$

where $r_{0}$ is a constant chosen to satisfy $\left\{\begin{array}{ll}r_{0}>2 m, & \text { if } m \geq 0 \\ r_{0}>0, & \text { if } m<0 .\end{array}\right.$ Here $m$ is the ADM mass of the Schwarzschild metric $g^{m}, r$ is the radial coordinate on $\left[r_{0}, \infty\right)$, and $d \sigma^{2}$ denotes the standard metric on the unit sphere $S^{2} \subset \mathbb{R}^{3}$.

Let $N$ be the positive function on $M_{r_{0}}^{m}$ defined by

$$
N=\sqrt{1-\frac{2 m}{r}}
$$


In terms of $N, g^{m}$ takes the form

$$
g^{m}=\frac{1}{N^{2}} d r^{2}+r^{2} d \sigma^{2} .
$$

The next lemma follows directly from the existence theory established in [14] (see also [3]).

Lemma 1. Let $\Sigma_{0}$ be the boundary of $\left(M_{r_{0}}^{m}, g^{m}\right)$. Given any positive function $\phi$ on $\Sigma_{0}$, there exists a positive function $u$ on $M_{r_{0}}^{m}$ such that

(i) The metric

$$
g_{u}^{m}=\left(\frac{u}{N}\right)^{2} d r^{2}+r^{2} d \sigma^{2}
$$

has zero scalar curvature and is asymptotically flat.

(ii) The mean curvature of $\Sigma_{0}$ (with respect to the $\infty$-pointing normal) in $\left(M_{r_{0}}^{m}, g_{u}^{m}\right)$ is equal to $\phi$.

(iii) The quotient $\frac{u}{N}$ has the asymptotic expansion

$$
\frac{u}{N}=1+\frac{m_{0}}{r}+O\left(\frac{1}{r^{2}}\right) \text { as } r \rightarrow \infty,
$$

where $m_{0}$ is the ADM mass of $g_{u}^{m}$.

Proof. Consider a Euclidean background metric

$$
d s^{2}=d r^{2}+r^{2} d \sigma^{2}
$$

on $M_{r_{0}}^{m}=S^{2} \times\left[r_{0}, \infty\right)$. By Theorem 2.1 in [14], there is a unique positive function $v$ on $M_{r_{0}}^{m}$ such that

$$
g_{v}=v^{2} d r^{2}+r^{2} d \sigma^{2}
$$

has zero scalar curvature, is asymptotically flat and the mean curvature of $\Sigma_{0}$ in $\left(M_{r_{0}}^{m}, g_{v}\right)$ is given by $\phi$. Furthermore, $v$ has an asymptotic expansion

$$
v=1+\frac{m_{0}}{r}+O\left(\frac{1}{r^{2}}\right),
$$

where $m_{0}$ is the ADM mass of $g_{v}$. Let $u=N v$, Lemma 1 is proved.

We note that metrics of the form $v^{2} d r^{2}+r^{2} d \sigma^{2}$ are called (shear free) quasi-spherical metrics [3]. By the formula (2.26) in [3] (or (1.10) in [14]), the differential equation satisfied by $v=u N^{-1}$ in Lemma 1 is

$$
\frac{2}{r} \frac{\partial v}{\partial r}=\frac{v^{2}}{r^{2}} \triangle_{S^{2}} v+\frac{\left(v-v^{3}\right)}{r^{2}}
$$

where $\triangle_{S^{2}}$ denotes the Laplacian operator of the metric $d \sigma^{2}$ on $S^{2}$. 
Proposition 1. Let $u, g_{u}^{m}, m_{0}$ be given in Lemma 1, Let $\Sigma_{r}$ be the radial coordinate sphere in $M_{r_{0}}^{m}$, i.e. $\Sigma_{r}=S^{2} \times\{r\}$. Let $H_{S}, H_{u}$ be the mean curvature of $\Sigma_{r}$ with respect to the metric $g^{m}, g_{u}^{m}$. Then

$$
\oint_{\Sigma_{r}} N\left(H_{S}-H_{u}\right) d \sigma
$$

is monotone non-increasing in $r$. Furthermore,

$$
\lim _{r \rightarrow \infty} \oint_{\Sigma_{r}} N\left(H_{S}-H_{u}\right) d \sigma=8 \pi\left(m_{0}-m\right) .
$$

Proof. We have $H_{S}=\frac{2}{r} N$ and $H_{u}=\frac{2}{r} v^{-1}$, where $v=u N^{-1}$. Hence

$$
\begin{aligned}
\oint_{\Sigma_{r}} N\left(H_{S}-H_{u}\right) d \sigma & =\oint_{\Sigma_{r}}\left(\frac{2}{r}\right)\left(N^{2}-N v^{-1}\right) d \sigma \\
& =\oint_{S^{2}} 2 r\left(N^{2}-N v^{-1}\right) d \omega,
\end{aligned}
$$

where $d \omega=r^{-2} d \sigma$ is the surface measure of $d \sigma^{2}$ on $S^{2}$. As $N^{2}=1-\frac{2 m}{r}$, we have

$$
\oint_{\Sigma_{r}} N\left(H_{S}-H_{u}\right) d \sigma=\oint_{S^{2}}\left(2 r-4 m-2 r N v^{-1}\right) d \omega .
$$

Therefore,

$$
\begin{aligned}
\frac{d}{d r} \oint_{\Sigma_{r}} N\left(H_{S}-H_{u}\right) d \sigma= & \oint_{S^{2}}\left[\left(2-2 N v^{-1}\right)-2 r \frac{\partial N}{\partial r} v^{-1}\right] d \omega \\
& +\oint_{S^{2}} 2 r N v^{-2} \frac{\partial v}{\partial r} d \omega .
\end{aligned}
$$

By (23), we have

$$
v^{-2} \frac{\partial v}{\partial r}=\frac{1}{2 r} \triangle_{S^{2}} v+\frac{\left(v^{-1}-v\right)}{2 r} .
$$

Thus the last term in (27) becomes

$$
\begin{aligned}
\oint_{S^{2}} 2 r N v^{-2} \frac{\partial v}{\partial r} d \omega & =\oint_{S^{2}} N \triangle_{S^{2}} v d \omega+\oint_{S^{2}} N\left(v^{-1}-v\right) d \omega \\
& =\oint_{S^{2}} N\left(v^{-1}-v\right) d \omega
\end{aligned}
$$

where we have used the fact that $N$ is a constant on each $\Sigma_{r}$ and $\oint_{S^{2}} \triangle_{S^{2}} v d \omega=0$. Hence the right side of (27) is given by

$$
\oint_{S^{2}}\left[\left(2-2 N v^{-1}\right)-2 r \frac{\partial N}{\partial r} v^{-1}+N\left(v^{-1}-v\right)\right] d \omega .
$$


Replace $v$ by $u N^{-1}$, the integrand of (30) becomes

$$
2-N^{2} u^{-1}-2 r \frac{\partial N}{\partial r} N u^{-1}-u \text {. }
$$

By (16), we have

$$
N^{2}+2 r N \frac{\partial N}{\partial r}=1
$$

Therefore, it follows from (27), (30), (31) and (32) that

$$
\frac{d}{d r} \oint_{\Sigma_{r}} N\left(H_{S}-H_{u}\right) d \sigma=-\oint_{S^{2}} u^{-1}(u-1)^{2} d \omega
$$

which proves that $\oint_{\Sigma_{r}} N\left(H_{S}-H_{u}\right) d \sigma$ is monotone non-increasing in $r$.

To evaluate $\lim _{r \rightarrow \infty} \oint_{\Sigma_{r}} N\left(H_{S}-H_{u}\right) d \sigma$, we have

$$
N v^{-1}=1-\frac{\left(m_{0}+m\right)}{r}+O\left(\frac{1}{r^{2}}\right)
$$

by (16) and (22). Therefore, by (26) we have

$$
\oint_{\Sigma_{r}} N\left(H_{S}-H_{u}\right) d \sigma=\oint_{S^{2}} 2\left(m_{0}-m\right) d \omega+O\left(r^{-1}\right),
$$

which implies

$$
\lim _{r \rightarrow \infty} \oint_{\Sigma_{r}} N\left(H_{S}-H_{u}\right) d \sigma=8 \pi\left(m_{0}-m\right) .
$$

Proposition 1 is proved.

3.2. Application of the Riemannian Penrose Inequality. In this section, we glue a body surrounding horizons, whose outer boundary is metrically a round sphere, to an asymptotically flat manifold $\left(M_{r_{0}}^{m}, g_{u}^{m}\right)$ constructed in Lemma 1, and apply the Riemannian Penrose Inequality and Proposition 1 to prove Theorem 1

We start with the following lemma.

Lemma 2. Let $\Omega$ be a body surrounding horizons. Suppose its outer boundary $\Sigma_{O}$ has positive mean curvature, then its horizon boundary $\Sigma_{H}$ strictly minimizes area among all closed surfaces in $\Omega$ that enclose $\Sigma_{H}$.

Proof. As $\Omega$ is compact and the mean curvature vector of $\Sigma_{O}$ points into $\Omega$, it follows from the standard geometric measure theory that there exist surfaces that minimize area among all closed surfaces in $\Omega$ that enclose $\Sigma_{H}$, furthermore none of the minimizers touches $\Sigma_{O}$. Let $\Sigma$ be any such a minimizer. By the Regularity Theorem 1.3 in [9], $\Sigma$ is a $C^{1,1}$ surface, and is $C^{\infty}$ where it does not touch $\Sigma_{H}$; moreover, the 
mean curvature of $\Sigma$ is 0 on $\Sigma \backslash \Sigma_{H}$ and equals the mean curvature of $\Sigma_{H} \mathcal{H}^{2}$-a.e. on $\Sigma \cap \Sigma_{H}$. Suppose $\Sigma$ is not identically $\Sigma_{H}$. As $\Sigma_{H}$ has zero mean curvature, the maximum principle implies that $\Sigma$ does not touch $\Sigma_{H}$. Hence, $\Sigma$ is a smooth closed minimal surface in the interior of $\Omega$, contradicting the assumption that $\Omega$ has no other closed minimal surfaces except $\Sigma_{H}$. Therefore, $\Sigma$ must be identically $\Sigma_{H}$.

Let $\Omega$ be a body surrounding horizons given in Theorem 1, Let $R$ and $R_{H}$ be the area radii of $\Sigma_{O}$ and $\Sigma_{H}$, which are defined by

$$
4 \pi R^{2}=\left|\Sigma_{O}\right| \text { and } 4 \pi R_{H}^{2}=\left|\Sigma_{H}\right| .
$$

It follows from Lemma 2 that $R>R_{H}$. To proceed, we choose $\left(M^{m}, g^{m}\right)$ to be one-half of a spatial Schwarzschild manifold whose horizon has the same area as $\Sigma_{H}$, i.e.

$$
\left(M^{m}, g^{m}\right)=\left(S^{2} \times\left[R_{H}, \infty\right), \frac{1}{1-\frac{2 m}{r}} d r^{2}+r^{2} d \sigma^{2}\right),
$$

where $m$ is chosen to satisfy $2 m=R_{H}$. As $R>R_{H}, \Sigma_{O}$ can be isometrically imbedded in $\left(M^{m}, g^{m}\right)$ as the coordinate sphere

$$
\Sigma_{R}=\{r=R\} \text {. }
$$

Henceforth, we identify $\Sigma_{O}$ with $\Sigma_{R}$ through this isometric imbedding. Let $M_{o}^{m}$ denote the exterior of $\Sigma_{O}$ in $M^{m}$. By Lemma 1 and Proposition 1, there exists a metric

$$
g_{u}^{m}=\left(\frac{u}{N}\right)^{2} d r^{2}+r^{2} d \sigma^{2}
$$

on $M_{o}^{m}$ such that $g_{u}^{m}$ has zero scalar curvature, is asymptotically flat, and the mean curvature of $\Sigma_{O}$ (with respect to the $\infty$-pointing normal) in $\left(M_{o}^{m}, g_{u}^{m}\right)$ agrees with $H$, the mean curvature of $\Sigma_{O}$ in $\Omega$. Furthermore, the integral

$$
\oint_{\Sigma_{r}} N\left(H_{S}-H_{u}\right) d \sigma
$$

is monotone non-increasing in $r$ and converges to $8 \pi\left(m_{0}-m\right)$ as $r \rightarrow \infty$, where $m_{0}$ is the ADM mass of $g_{u}^{m}$.

Now we attach this asymptotically flat manifold $\left(M_{o}^{m}, g_{u}^{m}\right)$ to the compact body $\Omega$ along $\Sigma_{O}$ to get a complete Riemannian manifold $M$ whose boundary is $\Sigma_{H}$. The resulting metric $g_{M}$ on $M$ satisfies the properties that it is asymptotically flat, has nonnegative scalar curvature away from $\Sigma_{O}$, is Lipschitz near $\Sigma_{O}$, and the mean curvatures of $\Sigma_{O}$ computed in both sides of $\Sigma_{O}$ in $M$ (with respect to the $\infty$ pointing normal) agree identically. 
Lemma 3. The horizon boundary $\Sigma_{H}$ is strictly outer minimizing in $M$, i.e $\Sigma_{H}$ strictly minimizes area among all closed surfaces in $M$ that enclose $\Sigma_{H}$.

Proof. By the construction of $g_{u}^{m}$, we know $\left(M_{o}^{m}, g_{u}^{m}\right)$ is foliated by $\left\{\Sigma_{r}\right\}_{r \geq R}$, where each $\Sigma_{r}$ has positive mean curvature. Let $\Sigma$ be a surface that minimizes area among surfaces in $M$ that encloses $\Sigma_{H}$ (such a minimizer exists as $M$ is asymptotically flat). We claim that $\Sigma \backslash \Omega$ must be empty, for otherwise $\Sigma \backslash \Omega$ would be a smooth, compact minimal surface in $\left(M_{o}^{m}, g_{u}^{m}\right)$ with boundary lying in $\Sigma_{O}$, and that would contradict the maximum principle. Therefore, $\Sigma \subset \Omega$. It then follows from Lemma 2 that $\Sigma=\Sigma_{H}$.

The next lemma is an application of the "corner smoothing" technique in [10].

Lemma 4. There exists a sequence of smooth asymptotically flat metrics $\left\{h_{k}\right\}$ defined on the background manifold of $M$ such that $\left\{h_{k}\right\}$ converges uniformly to $g_{M}$ in the $C^{0}$ topology, each $h_{k}$ has nonnegative scalar curvature, $\Sigma_{H}$ has zero mean curvature with respect to each $h_{k}$ (in fact $\Sigma_{H}$ can be made totally geodesic w.r.t $h_{k}$ ), and the ADM mass of $h_{k}$ converges to the ADM mass of $g_{M}$.

Proof. Let $M^{\prime}$ be an exact copy of $M$. We glue $M$ and $M^{\prime}$ along their common boundary $\Sigma_{H}$ to get a Riemannian manifold $\bar{M}$ with two asymptotic ends. Let $g_{\bar{M}}$ be the resulting metric on $\bar{M}$ and let $\Sigma_{O}^{\prime}$ be the copy of $\Sigma_{O}$ in $M^{\prime}$. Denote by $\Sigma$ the union of $\Sigma_{O}, \Sigma_{H}$ and $\Sigma_{O}^{\prime}$, we then know that the mean curvatures of $\Sigma$ computed in both sides of $\Sigma$ in $\bar{M}$ (with respect to normal vectors pointing to the same end of $\bar{M}$ ) agree. (At $\Sigma_{O}$ and $\Sigma_{O}^{\prime}$, this is guaranteed by the construction of $g_{u}^{m}$, and at $\Sigma_{H}$, this is provided by the fact that $\Sigma_{H}$ has zero mean curvature.)

Apply Proposition 3.1 in [10] to $\bar{M}$ at $\Sigma$, followed by a conformal deformation as described in Section 4.1 in [10], we get a sequence of smooth asymptotically flat metrics $\left\{g_{k}\right\}$, defined on the background manifold of $\bar{M}$, with nonnegative scalar curvature such that $\left\{g_{k}\right\}$ converges uniformly to $g_{\bar{M}}$ in the $C^{0}$ topology and the ADM mass of $g_{k}$ converges to the ADM mass of $g_{\bar{M}}$ on both ends of $\bar{M}$. Furthermore, as $\bar{M}$ has a reflection isometry (which maps a point $x \in M$ to its copy in $M^{\prime}$ ), detailed checking of the construction in Section 3 in [10] shows that $\left\{g_{k}\right\}$ can be produced in such a way that each $g_{k}$ also has the same reflection isometry. (Precisely, this can be achieved by choosing the mollifier $\phi(t)$ in equation (8) in [10] and the cut-off function $\sigma(t)$ in equation (9) in [10] to be both even functions.) Therefore, if we let 
$\bar{M}_{k}$ be the Riemannian manifold obtained by replacing the metric $g_{\bar{M}}$ by $g_{k}$ on $\bar{M}$, then $\Sigma_{H}$ remains a surface with zero mean curvature in $\bar{M}_{k}$ (in fact $\Sigma_{H}$ is totally geodesic). Define $h_{k}$ to be the restriction of $g_{k}$ to the background manifold of $M$, Lemma 4 is proved.

We continue with the proof of Theorem1, Let $\left\{h_{k}\right\}$ be the metric approximation of $g_{M}$ provided in Lemma 4. Let $M_{k}$ be the asymptotically flat manifold obtained by replacing the metric $g_{M}$ on $M$ by $h_{k}$. For any surface $\tilde{\Sigma}$ in $M$, let $|\tilde{\Sigma}|_{k},|\tilde{\Sigma}|$ be the area of $\tilde{\Sigma}$ w.r.t the induced metric from $h_{k}, g_{M}$ respectively. We can not apply the Riemannian Penrose Inequality directly to claim $m_{A D M}\left(h_{k}\right) \geq \sqrt{\frac{\left|\Sigma_{H}\right|_{k}}{16 \pi}}$. That is because we do not know if $\Sigma_{H}$ remains to be the outermost minimal surface in $M_{k}$. However, since $\Sigma_{H}$ is a minimal surface in $M_{k}$, we know the outermost minimal surface in $M_{k}$, denoted by $\Sigma_{k}$, exists and its area satisfies

$$
\left|\Sigma_{k}\right|_{k}=\inf \left\{|\tilde{\Sigma}|_{k} \mid \tilde{\Sigma} \in \mathcal{S}\right\}
$$

where $\mathcal{S}$ is the set of closed surfaces $\tilde{\Sigma}$ in $M$ that enclose $\Sigma_{H}$ (see [5], [9]). By the Riemannian Penrose Inequality (Theorem 1 in [5]), we have

$$
m_{A D M}\left(h_{k}\right) \geq \sqrt{\frac{\left|\Sigma_{k}\right|_{k}}{16 \pi}} .
$$

Let $k$ approach infinity, we have

$$
\lim _{k \rightarrow \infty} m_{A D M}\left(h_{k}\right)=m_{A D M}\left(g_{M}\right),
$$

and

$$
\lim _{k \rightarrow \infty}\left|\Sigma_{k}\right|_{k}=\inf \{|\tilde{\Sigma}| \mid \tilde{\Sigma} \in \mathcal{S}\}
$$

where we have used (42) and the fact that $\left\{h_{k}\right\}$ converges uniformly to $g_{M}$ in the $C^{0}$ topology. By Lemma 3, we also have

$$
\left|\Sigma_{H}\right|=\inf \{|\tilde{\Sigma}| \mid \tilde{\Sigma} \in \mathcal{S}\}
$$

Therefore, it follows from (43), (44), (45) and (46) that

$$
m_{A D M}\left(g_{M}\right) \geq \sqrt{\frac{\left|\Sigma_{H}\right|}{16 \pi}} .
$$

To finish the proof of Theorem 1, we make use of the monotonicity of the integral

$$
\oint_{\Sigma_{r}} N\left(H_{S}-H_{u}\right) d \sigma .
$$


By Proposition 1, we have

$$
\begin{aligned}
\oint_{\Sigma_{O}} N\left(H_{S}-H_{u}\right) d \sigma & \geq \lim _{r \rightarrow \infty} \oint_{\Sigma_{r}} N\left(H_{S}-H_{u}\right) d \sigma \\
& =8 \pi\left(m_{0}-m\right) .
\end{aligned}
$$

On the other hand, we know

$$
m_{0}=m_{A D M}\left(g_{u}^{m}\right)=m_{A D M}\left(g_{M}\right),
$$

and

$$
m=\frac{1}{2} R_{H}=\sqrt{\frac{\left|\Sigma_{H}\right|}{16 \pi}} .
$$

Therefore, it follows from (49), (50), (51) and (47) that

$$
\oint_{\Sigma_{O}} N\left(H_{S}-H_{u}\right) d \sigma \geq 0
$$

Plug in $H_{S}=\frac{2}{R} N, H_{u}=H$ and $N=\sqrt{1-\frac{R_{H}}{R}}$, we then have

$$
8 \pi R \sqrt{1-\frac{R_{H}}{R}} \geq \oint_{\Sigma_{O}} H d \sigma .
$$

Direct computation shows that (153) is equivalent to (31). Hence, (3) is proved.

Finally, when the equality in (3) holds, we have

$$
\oint_{\Sigma_{r}} N\left(H_{S}-H_{u}\right) d \sigma=0, \quad \forall r \geq R .
$$

By the derivative formula (33), $u$ is identically 1 on $M_{o}^{m}$. Therefore, the metric $g_{u}^{m}$ is indeed the Schwarzschild metric $g^{m}$. Since the mean curvature of $\Sigma_{O}$ in $\left(M_{o}^{m}, g_{u}^{m}\right)$ was arranged to equal $H$, the mean curvature of $\Sigma_{O}$ in $\Omega$, we conclude that $H=\frac{2}{R}\left(1-\frac{R_{H}}{R}\right)^{\frac{1}{2}}$, which is a constant. Theorem 1 is proved.

Comparing to the equality case in Theorem 2, one would expect that the equality in (3) holds if and only if $\Omega$ is isometric to a region, in a spatial Schwarzschild manifold, which is bounded by a rotationally symmetric sphere and the Schwarzschild horizon. We believe that this is true, but are not able to prove it at this stage. A confirmation of this expectation seems to require a good knowledge of the behavior of a sequence of asymptotically flat 3-manifolds with controlled $C^{0}$ geometry, on which the equality of the Riemannian Penrose Inequality is nearly satisfied. We leave this as an open question. 


\section{Some Discussion}

Let $\Sigma$ be an arbitrary closed 2-surface in a general 3-manifold $M$ with nonnegative scalar curvature. Consider the quantity

$$
m(\Sigma)=\sqrt{\frac{|\Sigma|}{16 \pi}}\left[1-\frac{1}{16 \pi|\Sigma|}\left(\oint_{\Sigma} H\right)^{2}\right]
$$

where $|\Sigma|$ is the area of $\Sigma, H$ is the mean curvature of $\Sigma$ in $M$ and we omit the surface measure $d \sigma$ in the integral. Theorem 1 suggests that, if $\Sigma$ is metrically a round sphere, $m(\Sigma)$ may potentially agree with a hidden definition of quasi-local mass of $\Sigma$. Such a speculation could be further strengthened by the resemblance between $m(\Sigma)$ and the Hawking quasi-local mass [8]

$$
m_{H}(\Sigma)=\sqrt{\frac{|\Sigma|}{16 \pi}}\left[1-\frac{1}{16 \pi} \oint_{\Sigma} H^{2}\right] .
$$

By Hölder's inequality, we have

$$
m(\Sigma) \geq m_{H}(\Sigma)
$$

for any surface $\Sigma$. On the other hand, if $\Sigma$ is a closed convex surface in the Euclidean space $\mathbb{R}^{3}$, the classic Minkowski inequality [12]

$$
\left(\oint_{\Sigma} H d \sigma\right)^{2} \geq 16 \pi|\Sigma|
$$

implies that $m(\Sigma) \leq 0$ and $m(\Sigma)=0$ if and only if $\Sigma$ is a round sphere in $\mathbb{R}^{3}$. Therefore, even though bigger than $m_{H}(\Sigma), m(\Sigma)$ shares the same character as $m_{H}(\Sigma)$ that it is negative on most convex surfaces in $\mathbb{R}^{3}$.

In order to gain positivity and to maintain the same numerical value on metrically round spheres, we propose to modify $m(\Sigma)$ in a similar way as the Brown-York mass $m_{B Y}(\Sigma)$ [6] is defined. Recall that, for those $\Sigma$ with positive Gaussian curvature, $m_{B Y}(\Sigma)$ is defined to be

$$
m_{B Y}(\Sigma)=\frac{1}{8 \pi}\left(\oint_{\Sigma} H_{0} d \sigma-\oint_{\Sigma} H d \sigma\right)
$$

where $H_{0}$ is the mean curvature of $\Sigma$ when it is isometrically embedded in $\mathbb{R}^{3}$. Now suppose $\Sigma$ is metrically a round sphere, then

$$
\left(\oint_{\Sigma} H_{0}\right)^{2}=16 \pi|\Sigma|
$$


In this case, we can re-wriite $m(\Sigma)$ as either

$$
m(\Sigma)=\sqrt{\frac{|\Sigma|}{16 \pi}}\left[1-\left(\frac{\oint_{\Sigma} H}{\oint_{\Sigma} H_{0}}\right)^{2}\right]
$$

or

$$
m(\Sigma)=\frac{1}{16 \pi}\left(\oint_{\Sigma} H_{0}\right)\left[1-\left(\frac{\oint_{\Sigma} H}{\oint_{\Sigma} H_{0}}\right)^{2}\right] .
$$

This motivates us to consider the following two quantities:

Definition 1. For any $\Sigma$ with positive Gaussican curvature, define

$$
m_{1}(\Sigma)=\sqrt{\frac{|\Sigma|}{16 \pi}}\left[1-\left(\frac{\oint_{\Sigma} H}{\oint_{\Sigma} H_{0}}\right)^{2}\right],
$$

and

$$
m_{2}(\Sigma)=\frac{1}{16 \pi}\left(\oint_{\Sigma} H_{0}\right)\left[1-\left(\frac{\oint_{\Sigma} H}{\oint_{\Sigma} H_{0}}\right)^{2}\right],
$$

where $H$ is the mean curvature of $\Sigma$ in $M$ and $H_{0}$ is the mean curvature of $\Sigma$ when it is isometrically embedded in $\mathbb{R}^{3}$.

The following result compares $m_{H}(\Sigma), m_{1}(\Sigma), m_{2}(\Sigma)$ and $m_{B Y}(\Sigma)$.

Theorem 3. Suppose $\Sigma$ is a closed 2-surface with positive Gaussian curvature in a 3-manifold $M$. Then

(i) $m_{1}(\Sigma) \geq m_{H}(\Sigma)$, and equality holds if and only if $\Sigma$ is metrically a round sphere and $\Sigma$ has constant mean curvature.

(ii) $m_{B Y}(\Sigma) \geq m_{2}(\Sigma)$, and equality holds if and only if $\oint_{\Sigma} H_{0} d \sigma=$ $\oint_{\Sigma} H d \sigma$.

(iii) Suppose $\Sigma$ bounds a domain $\Omega$ with nonnegative scalar curvature and the mean curvature of $\Sigma$ in $\Omega$ is positive, then

$$
m_{2}(\Sigma) \geq m_{1}(\Sigma) \geq 0
$$

Moreover, $m_{1}(\Sigma)=0$ if and only if $\Omega$ is isometric to a domain in $\mathbb{R}^{3}$, and $m_{2}(\Sigma)=m_{1}(\Sigma)$ if and only if either $\Omega$ is isometric to a domain in $\mathbb{R}^{3}$ in which case $m_{2}(\Sigma)=m_{1}(\Sigma)=0$ or $\Sigma$ is metrically a round sphere.

Proof. (i) Let $m(\Sigma)$ be defined as in (55). By the Minkowski inequality (58), we have $m_{1}(\Sigma) \geq m(\Sigma)$. By (57), we have $m(\Sigma) \geq m_{H}(\Sigma)$. Therefore, $m_{1}(\Sigma) \geq m_{H}(\Sigma)$ and equality holds if and only if $\Sigma$ is metrically a round sphere and the mean curvature of $\Sigma$ in $M$ is a constant. 
(ii) This case is elementary. Let $a=\oint_{\Sigma} H$ and $b=\oint_{\Sigma} H_{0}$. Then (ii) is equivalent to the inequality $\left(1-\frac{a}{b}\right)^{2} \geq 0$.

(iii) By the result of Shi and Tam [14, i.e. Theorem 2, we have

$$
1-\left(\frac{\oint_{\Sigma} H}{\oint_{\Sigma} H_{0}}\right)^{2} \geq 0
$$

with equality holding if and only if $\Omega$ is isometric to a domain in $\mathbb{R}^{3}$. (iii) now follows directly from (65) and the Minkowski inequality (58).

Suppose $\Omega$ is a compact 3-manifold with boundary with nonnegative scalar curvature and its boundary $\partial \Omega$ has positive Gaussian curvature and positive mean curvature. Theorem 3 implies that

$$
m_{B Y}(\partial \Omega) \geq m_{2}(\partial \Omega) \geq m_{1}(\partial \Omega) \geq m_{H}(\partial \Omega)
$$

with $m_{1}(\partial \Omega) \geq 0$ and $m_{B Y}(\partial \Omega)=m_{H}(\partial \Omega)$ if and only if $\Omega$ is isometric to a round ball in $\mathbb{R}^{3}$. This provides a slight generalization of a previous result of Shi and Tam (Theorem 3.1 (b) in [15]), which showed $m_{B Y}(\partial \Omega) \geq m_{H}(\partial \Omega)$.

\section{REFERENCES}

[1] R. Arnowitt, S. Deser, and C. W. Misner. Coordinate invariance and energy expressions in general relativity. Phys. Rev. (2), 122:997-1006, 1961.

[2] Robert Bartnik. New definition of quasilocal mass. Phys. Rev. Lett., 62(20):2346-2348, 1989.

[3] Robert Bartnik. Quasi-spherical metrics and prescribed scalar curvature. J. Differential Geom., 37(1):31-71, 1993.

[4] Hubert L. Bray. The Penrose inequality in general relativity and volume comparison theorems involving scalar curvature. Stanford University Thesis, 1997.

[5] Hubert L. Bray. Proof of the Riemannian Penrose inequality using the positive mass theorem. J. Differential Geom., 59(2):177-267, 2001.

[6] J. David Brown and James W. York, Jr. Quasilocal energy in general relativity. In Mathematical aspects of classical field theory (Seattle, WA, 1991), volume 132 of Contemp. Math., pages 129-142. Amer. Math. Soc., Providence, RI, 1992.

[7] D. Christodoulou and S.-T. Yau. Some remarks on the quasi-local mass. In Mathematics and general relativity (Santa Cruz, CA, 1986), volume 71 of Contemp. Math., pages 9-14. Amer. Math. Soc., Providence, RI, 1988.

[8] Stephen Hawking. Gravitational radiation in an expanding universe. J. Mathematical Phys., 9:598-604, 1968.

[9] Gerhard Huisken and Tom Ilmanen. The inverse mean curvature flow and the Riemannian Penrose inequality. J. Differential Geom., 59(3):353-437, 2001.

[10] Pengzi Miao. Positive mass theorem on manifolds admitting corners along a hypersurface. Adv. Theor. Math. Phys., 6(6):1163-1182 (2003), 2002.

[11] Louis Nirenberg. The Weyl and Minkowski problems in differential geometry in the large. Comm. Pure Appl. Math., 6:337-394, 1953. 
[12] G. Pólya and G. Szegö. Isoperimetric Inequalities in Mathematical Physics. Annals of Mathematics Studies, no. 27. Princeton University Press, Princeton, N. J., 1951.

[13] Richard Schoen and Shing Tung Yau. On the proof of the positive mass conjecture in general relativity. Comm. Math. Phys., 65(1):45-76, 1979.

[14] Yuguang Shi and Luen-Fai Tam. Positive mass theorem and the boundary behaviors of compact manifolds with nonnegative scalar curvature. J. Differential Geom., 62(1):79-125, 2002.

[15] Yuguang Shi and Luen-Fai Tam. Quasi-local mass and the existence of horizons. Comm. Math. Phys., 274(2):277-295, 2007.

[16] Edward Witten. A new proof of the positive energy theorem. Comm. Math. Phys., 80(3):381-402, 1981.

School of Mathematical Sciences, Monash University, Victoria, 3800, Australia.

E-mail address: Pengzi.Miao@sci.monash.edu.au 